\title{
Extracorporeal Membrane Oxygenation for Pulmonary Embolism During Pregnancy and Postpartum
}

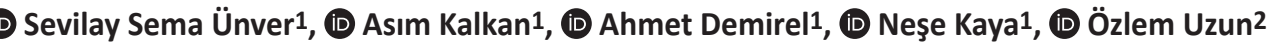 \\ 1 University of Health Sciences Turkey, Istanbul Okmeydanı Training and Research Hospital, Clinic of Emergency Medicine, Istanbul, Turkey \\ 2 istanbul Bağcılar Training and Research Hospital, Department of Emergency Medicine, istanbul, Turkey
}

\section{Abstract}

The maternal and fetal mortality rate in unstable pulmonary embolisms accompanied by obstructive cardiac shock is approximately $30 \%$. An intervention aimed at the pulmonary clot is essential under these conditions. There are various ways of removing this clot from the pulmonary arteries, including thrombolytic administration, percutaneous catheter-based thrombectomy, and direct surgical clot removal. These procedures all have advantages and disadvantages. Increasing use is being made of extracorporeal membrane oxygenation (ECMO) as a means of reducing these risks to a minimum. There are studies recommending ECMO together with thrombolysis or surgical thrombectomy. However, which study is superior to another is unclear. The purpose of the present study was, therefore, to assess the benefits and risks of ECMO use in pregnant patients with pulmonary embolism and to endeavor to produce a new algorithm.

Keywords: Extracorporeal membrane oxygenation, ECMO, pulmonary embolism, pregnancy, peripartum, postpartum

\section{INTRODUCTION}

Although pulmonary embolism is seen in between 1/1000 and $1 / 3000$ pregnancies, it is responsible for $5 \%$ of all mother and baby deaths. The maternal and fetal mortality rate in unstable pulmonary embolisms accompanied by obstructive cardiac shock is approximately 30\%. An intervention aimed at the pulmonary clot is essential under these conditions (1-3). There are various ways of removing this clot from the pulmonary arteries, including thrombolytic administration, percutaneous catheter-based thrombectomy, and direct surgical clot removal. These procedures all have advantages and disadvantages. Separate advice exists for each procedure in pregnant women. Studies have reported that thrombolytic administration increases the risk of bleeding. The peripartum and postpartum risks of bleeding differ. Case reports have shown an increased risk of bleeding following administration of thrombolysis to patients scheduled for cesarean delivery in particular (4-6).
Catheter-based thrombectomy of surgical thrombectomy can be performed in patients in whom thrombolysis is thought to be impossible or high-risk. Increasing use is being made of extracorporeal membrane oxygenation (ECMO) as a means of reducing these risks to a minimum. There are studies recommending ECMO together with thrombolysis or surgical thrombectomy. However, which study is superior to another is unclear. Considering maternal and fetal complications, there exists no specific algorithm concerning whether thrombolysis or thrombectomy should be performed before or during ECMO. At the same time, the literature consists solely of case reports, and there are no clinical studies involving large numbers of patients. Although there is one systematic review study of the management of pulmonary embolism in pregnant women, no studies have investigated ECMO use by itself (7). The purpose of the present study was, therefore, to assess the benefits and risks of ECMO use in pregnant patients with pulmonary embolism and to endeavor to produce a new algorithm. 


\section{ECMO}

ECMO use has increased markedly in recent years. Despite being an invasive method, frequent reports of clinical success have encouraged interest in its use. Mechanical ventilation and conservative fluid therapy are available to protect the lung in the traditional treatment of Acute Respiratory Distress syndrome (ARDS). ECMO is used to protect against the deleterious effects of the ventilator in refractory cases incapable of treatment. It provides temporary respiratory support until the lung damage resolves (by providing a period of "lung rest") (8). When the patient receives ECMO support, the clinician can lower both tidal volume and high oxygen concentrations fraction of inspired oxygen. The important thing is that the underlying disease in the patient scheduled for ECMO should be reversible (9). Two main methods are currently employed in clinical practice, and these are briefly discussed below.

\section{Veno-Venous (VV) ECMO}

The decision concerning when a patient should receive ECMO is complex. VV-ECMO should not be used in every patient with ARDS, and the fact it delays 'rescue therapies' in the course of the disease may limit its benefits. Patients indicated for VV-ECMO are shown in Table 1, but none of these represent absolute criteria. Experience clinicians must individualize patient selection (10).

In the application of ECMO, the majority of modern units employ a dual lumen cannula inserted inside the right jugular vein. One distal and one proximal lumen (both constituting a "drainage lumen") drain blood into the extracorporeal circuit from the inferior and superior vena cava. Oxygenated blood is subsequently returned from a second lumen (in the same cannula, in the middle of the catheter), through which the blood flows into the right atrium and then into the right ventricle (facing the tricuspid valve). The location and position of the cannula must be confirmed using echocardiography in order to avoid malpositioning. Blood flows in the circuit through an oxygenator and a temperature regulator that warms the blood before returning to the body. Oxygenation is set with a blood flow rate in the circuit (generally between 3 and $4 \mathrm{~L} / \mathrm{min}$ ) and an oxygen fraction generally set at $1.0(100 \%)$. Clean air and oxygen are mixed through a semi-porous membrane in a mixer before the gas is exposed to blood. Carbon dioxide $\left(\mathrm{CO}_{2}\right)$ removal is independent of blood flow (11). Since both inflammatory and clotting systems are activated by contact with the ECMO circuit, fractionated heparin anticoagulation is generally required to prevent clotting (12). At the same time, other interventions, such as aggressive diuresis and antibiotics (in case of sepsis), must be provided in order to optimize pulmonary functions. ECMO can easily be stopped once lung functions improve. When this happens, blood flows easily from the circuit, but no gas exchange takes place. If sufficient oxygenation and ventilation are achieved after a few hours' observation, the cannula can then be removed. Unsurprisingly, therapeutic evidence in the pregnant population is very limited. The use of $\mathrm{VV}$-ECMO in the treatment of severe ARDS during pregnancy and postnatally first appeared in the H1N1 epidemic of 2009. Observational data showed that ECMO might be beneficial in pregnant patients with ARDS secondary to H1N1 infection. One recent meta-analysis concluded that there is a $75 \%$ survival rate in pregnant patients receiving VV-ECMO for ARDS secondary to H1N1. However, there are no data comparing ECMO with respiration preserving mechanical ventilation in the same patient group. To summarize, there are no data supporting the usefulness of VV-ECMO for severe ARDS in any sub-population (13-16).

\section{Veno-Arterial (VA) ECMO}

VA-ECMO can provide support in patients with both respiratory distress and severe cardiac problems. These indications may be listed as cardiac arrest due to reversible causes (such as drug intoxications), refractory cardiogenic shock (such as peripartum cardiomyopathy and myocardial infarction), cardiopulmonary bypass failure, or pulmonary embolism (17). The precipitating factor should be reversible in deciding on whether pregnant patients are suitable for VA-ECMO (Table 2).

In clinical practice, peripheral cannulation is more widely employed for VA-ECMO. Venous blood is generally drained via a cannula inserted in the femoral vein, and the flow is diverted to the $\mathrm{EC}<0$ circuit. Similarly to $\mathrm{VV}$-ECMO, $\mathrm{CO}_{2}$ removal depends on the sweep gas flow and oxygenation pump flow set by the operator. Oxygenated blood obtained from the venous system is most commonly transferred to the arterial system through a catheter installed in the femoral artery. This permits blood oxygenation (Figure 1) $(18,19)$.

\begin{tabular}{|l|}
\hline Table 1. Probable indications for gestational and postpartum VV-ECMO support \\
\hline Severe (but potentially reversible) respiratory failure \\
\hline Severe respiratory acidosis and hypercapnia despite respiratory rate increasing to 35/minute with mechanical ventilation \\
\hline $\mathrm{FiO}_{2} \geq 0.9$ and $\mathrm{PEEP} \geq 10 \mathrm{~cm} \mathrm{H}_{2} \mathrm{O}$ and a $\mathrm{PaO}_{2} / \mathrm{FiO}_{2}$ ration less than 100 despite ventilator support and the use of normal support techniques \\
\hline $\mathrm{VV}$ : Veno-venous, ECMO: Extracorporeal membrane oxygenation, FiO ${ }_{2}$ : Fraction of inspired oxygen PEEP: Positive end expiratory pressure, PaO ${ }_{2}$ : Partial oxygen pressure \\
\hline
\end{tabular}


Appropriate keywords were scanned up to 31 December 2019, in English on PubMed, Clinical Key/Elsevier, EBSCO Discovery Service, MD Consult Science Direct, Scopus, EMBASE, Medscape, and Google Scholar electronic search engines for data collection. The search criteria involved the keywords "pregnancy", "pregnancy and pulmonary embolism", "ECMO", and "pregnancy-pulmonary embolism and ECMO". Publications in languages other than English were not included in this study. We detected 2819 studies using the keywords pregnancy and pulmonary embolism published between 1947 and 2020. These consisted of 575 review studies, four book chapters, 34 clinical studies, 14 meta-analysis, and 11 randomized controlled studies. The remainders were other types of publications, particularly case reports. When

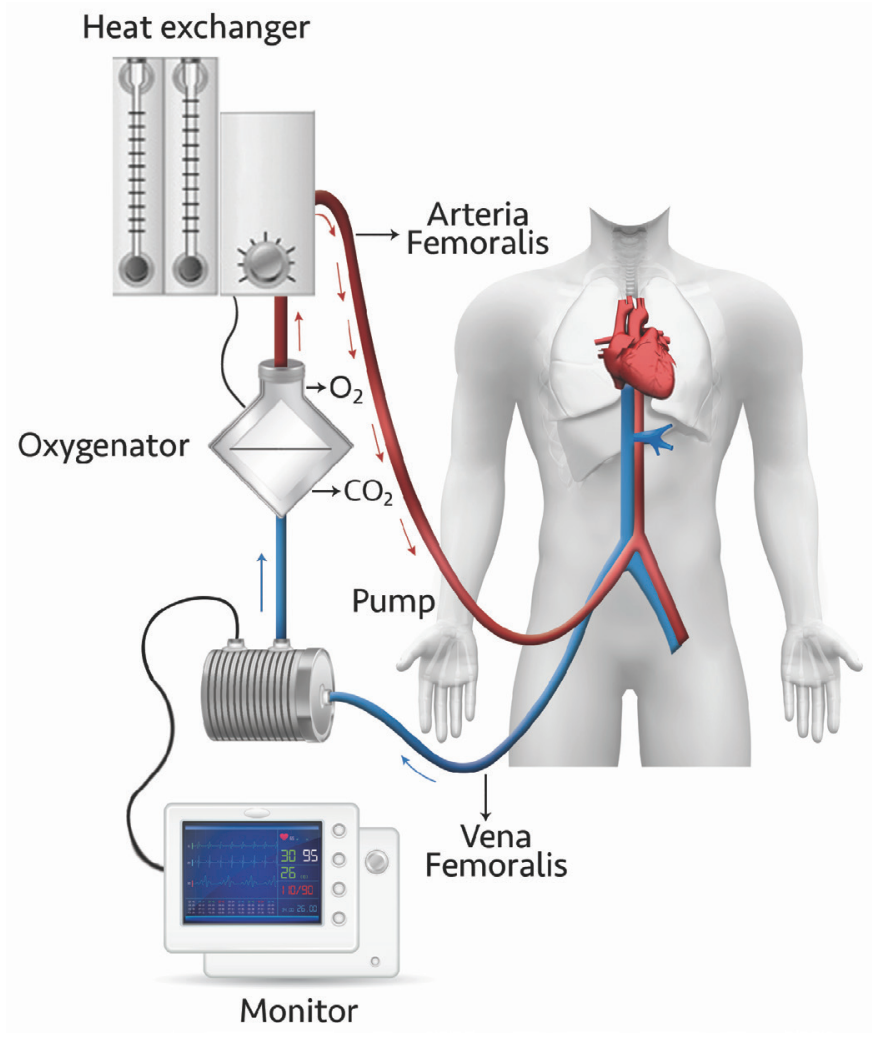

Figure 1. Demonstration of extracorporeal membrane oxygenation we added ECMO to the keywords pregnancy and pulmonary embolism, the number of studies identified declined to 21. One, a case report, described a 27-year-old woman with uterine arteriovenous malformation, and since the patient was not pregnant, the publication was not included in this study (20). Another publication concerned a patient initially thought to have pulmonary embolism but subsequently diagnosed with pre-eclampsia and undergoing ECMO. That report was also excluded from the present study (21). Another case report involving peripartum heart failure and ECMO application was also excluded (22). One of the publications we identified concerned a case report of respiratory insufficiency developing after influence, and another concerned ECMO us in a pregnant patient developing acute lung damage. Another case report concerned a pregnant woman with amniotic fluid embolism. There were also four review studies. All these concerned the use of ECMO in respiratory insufficiency in pregnant women, and one also concerned pulmonary embolism and ECMO use in pregnancy $(7,23,24)$.

Once all these publications had been excluded, we identified nine case reports compatible with the scope of our review. These case reports involved ten patients. The number of cases was very small, and we, therefore, deepened our research to examine the content of cases of pulmonary embolism and pregnancy in greater detail. The reviews were examined in terms of content. Three pulmonary embolisms were identified in patients undergoing ECMO due to respiratory insufficiency. Examination of the references to these pulmonary embolism cases showed that they were the same as the cases included in our study. The references of the case reports included in our study were then examined, and no different cases were encountered.

The case reports included in our study were examined, and patients' ages, time of ECMO application (peripartum or postpartum), gestational weeks, anticoagulation applied before ECMO, and thrombolytic conditions or thrombectomy were recorded. Mother and baby survival was also recorded.

\begin{tabular}{|l|}
\hline Table 2. Probable indications for gestational and postpartum VA-ECMO support \\
\hline Peripartum cardiomyopathy-related refractory left ventricular failure, myocardial infarction, myocarditis \\
\hline Drug intoxication requiring prolonged cardiopulmonary resuscitation \\
\hline Refractory right or left ventricular failure in cases of suspected amniotic fluid embolism \\
\hline Cardiopulmonary bypass failure following heart surgery \\
\hline Massive pulmonary embolism with refractory failure in the right ventricle \\
\hline Prolonged cardiopulmonary resuscitation due to a potentially reversible cause \\
\hline VA: Veno-arterial, ECMO: Extracorporeal membrane oxygenation \\
\hline
\end{tabular}


Only nine of the 2891 publications identified could be included in our study. These nine case reports involved a total of 10 patients. The pregnant women in the nine case reports were aged between 21 and 37 years. Six were in the third trimester of pregnancy, three in the second, and one in the first. A cardiopulmonary arrest occurred in the emergency department in seven pregnant women, and acute right heart failure-related shock in three. VA-ECMO was applied in all patients, except for one. However, due to the development of Compartment syndrome associated with bleeding in the upper extremity, VV-ECMO had to be performed subsequently on two patients. Four patients underwent thrombectomy, and four received thrombolytic. Cather-directed thrombolysis was administered to one of the patients. Only one patient received ECMO together with unfractionated heparin. Anticoagulant therapy was only not administered to one of the patients. That patient received thrombolysis with tenecteplase, and ECMO was performed. Five patients received heparin for anticoagulation, and four received low molecular weight heparin. Almost all patients underwent an emergency cesarean section. Abortion was performed on one patient in the first trimester and another in the second (Table 3).

Vaginal, tracheal, and intra-abdominal bleeding occurred in three patients receiving thrombolysis before the cesarean section. Compartment syndrome associated with bleeding in the radial artery subjected to catheterization also occurred in one of these patients. Acute kidney failure associated with intra-abdominal bleeding occurred in one of the two patients receiving thrombolysis immediately after cesarean section, while thrombosis in the femoral vein subjected to catheterization and Compartment syndrome associated with bleeding in the radial artery developed in the other (Table 4).

Vaginal bleeding, tracheal bleeding, uterine artery hemorrhage, and acute kidney failure developed as complications in three of the four patients undergoing thrombectomy following a cesarean. However, three of these had also previously undergone thrombolysis. One patient underwent thrombectomy only, and no complication developed. ECMO was applied for at least 60 hours and a maximum of seven days (Table 4). All patients were discharged. Only two patients were discharged with neurological sequelae, while the others were all discharged in a healthy condition. (25-32)

\section{DISCUSSION}

Our detailed examination of the literature revealed nine cases of pulmonary embolism undergoing ECMO. No maternal death was reported in these cases. Two baby deaths occurred in pregnancy. The fact that all patients receiving ECMO, irrespective of thrombolysis or thrombectomy, appears to show that ECMO is a good method in pregnant patients with pulmonary embolism. The presence of postpartum hemorrhages in our patients receiving thrombolysis may encourage ECMO to be used together with percutaneous or surgical thrombectomy. This technique may be suitable for avoiding massive postpartum bleeding associated with

Table 3. Characteristics and outcomes, stratified by treatment modalities

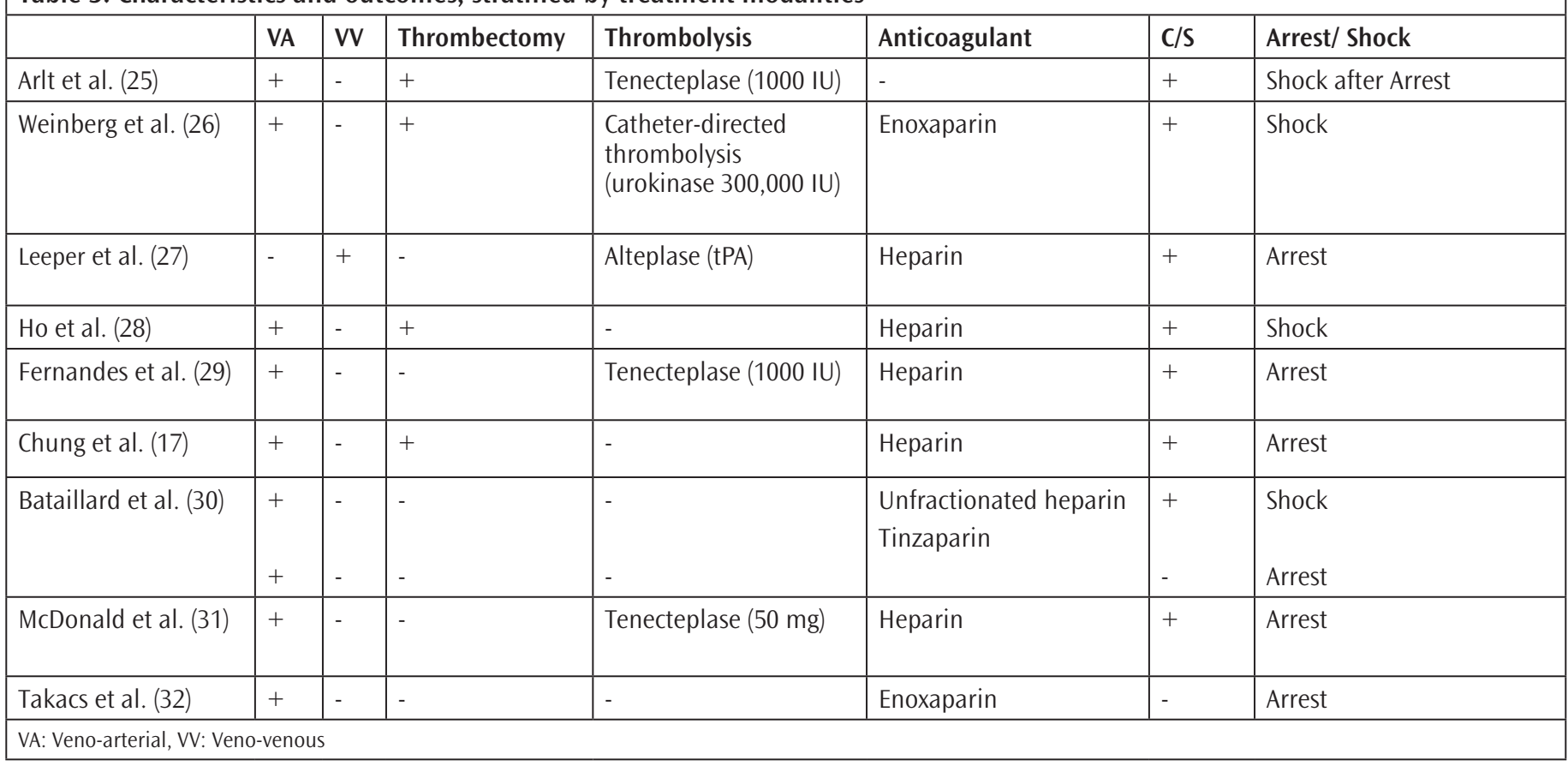


thrombolysis, particularly after delivery. No complications occurred in the three patients not receiving thrombolysis in this review. ECMO was applied in one of these patients following catheter-directed thrombectomy. Chung et al. (17) applied ECMO after thrombectomy in their case reports, and subsequently administered heparin infusion. They suggested that major complications such as hemorrhage could be avoided with this method. Martilotti et al. (7) made a similar suggestion in their systematic review. Those authors determined a survival rate of $93.8 \%$ in 36 pregnant patients undergoing surgical thrombectomy due to pulmonary embolism. They detected major bleeding in only seven of these patients and reported that this was a thrombectomy complication. Although our data are very limited, the use of ECMO together with thrombectomy can improve survival in pregnant patients. All patients receiving thrombolysis were individuals with cardiopulmonary arrest. Tenecteplase was given to all these patients. According to the 2015 cardiopulmonary resuscitation algorithm, thrombolysis should be administered if pulmonary embolism is suspected in pregnant women with cardiopulmonary arrest (33). This may be the main reason for thrombolysis being given to these patients. Despite the limited number of patients, our findings also show that thrombolytic therapy should be administered if cardiopulmonary arrest associated with pulmonary embolism occurs in a pregnant woman. If there is no arrest, but findings of acute right heart failure are present, then percutaneous or surgical thrombectomy represents a good option.
VV-ECMO is more used in respiratory failure in pregnant patients. Moore et al. (11) investigated ECMO use in pregnant patients and found that VA-ECMO was applied to only four out of 45 patients. They determined general maternal and fetal survival rates of $77.8 \%$ with VV-ECMO and 65\% with VA-ECMO. Based on these data, they suggested that VV-ECMO was superior. That study contains no definitive information concerning pulmonary embolism-related ECMO use. In the present review, VV-ECMO was applied to only one patient. This was then switched to VAECMO to increase cardiac support once spontaneous circulation had been established. Our findings directly contradict Moore et al. (11) suggestion. This is because Moore et al. (11) study contained no pulmonary embolism patients, while the present review considered only pregnant patients with pulmonary embolism. Based on our existing data, we may conclude that VAECMO is more beneficial in pregnant patients with pulmonary embolism. Bleeding and associated Compartment syndrome occurred around the radial artery in two patients undergoing VA-ECMO. Artery selection was modified to the subclavian artery in these patients. This bleeding may be due to thrombolysis or anticoagulant use. Care must be taken in terms of Compartment syndrome development during ECMO with peripheral vein and artery cannulation. Such complications have been seen less frequently in recent years since the biocompatibility of ECMO has been enhanced (34).

There are several strengths to this review. In particular, we investigated several databases and examined all the review studies we encountered. We then investigated pregnant women

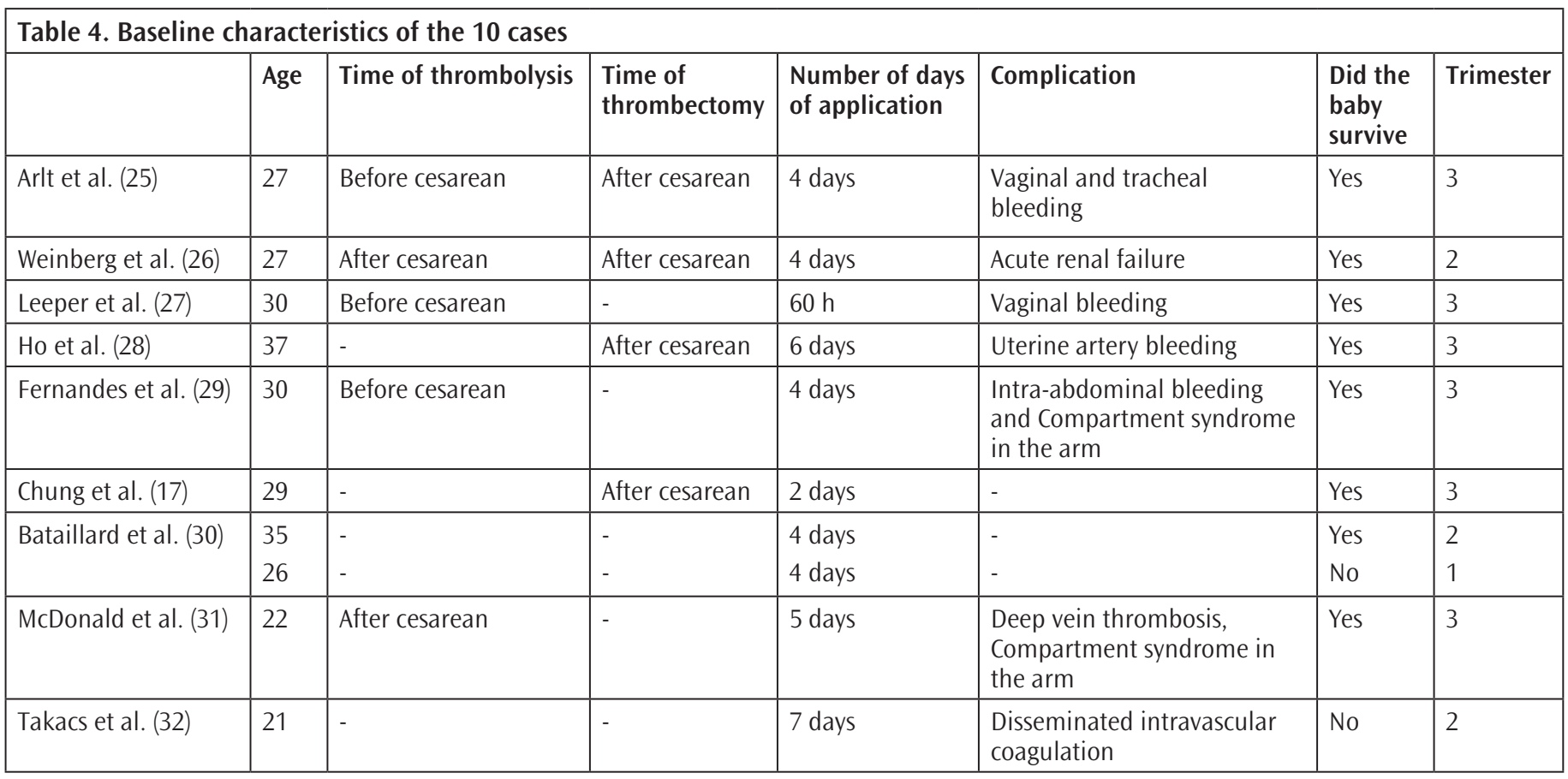


with pulmonary embolism from these reviews. We also checked the references of all the texts we were able to access.

Based on our findings, all patients survived, and no mortality occurred in any case. This also suggested that the case reports might have been biased. The absence of mortality among pregnant women with pulmonary embolism undergoing ECMO may derive from the author thinking that the report would not be capable of publication in any journal. This may be due to false high survival rates. Studies involving large case numbers in a clinic where the procedure is performed will be instructive for the future.

\section{CONCLUSION}

In conclusion, although we were unable to produce a definite algorithm with the very low patient number involved, we can list the procedures performed. When pulmonary embolism is suspected in pregnant patients, the presence or absence of cardiopulmonary arrest or acute right heart failure must be investigated. Thrombolysis appears beneficial during cardiopulmonary resuscitation in arrest patients. Emergency cesarean surgery must also be performed to avoid fetal mortality. ECMO must be performed once these procedures have been carried out. VA-ECMO appears to be a preferable option. Care must also be taken over the side effects of thrombolytic drugs in these patients. The duration of ECMO ranges between 60 hour and seven days, the essential factor being the complete disappearance of findings of right heart failure. Surgical or percutaneous thrombectomy may be beneficial in all patients. The best means of reducing bleeding complications appears to be ECMO together with thrombectomy. The best way of supporting all our conclusions will be through future studies involving more patients in this group undergoing ECMO.

\section{Ethics}

Peer-review: Internally peer-reviewed.

\section{Authorship Contributions}

Concept: A.K., S.S.Ü., Design: A.K., N.K., A.D., Data Collection or Processing: A.D., Ö.U., A.K., S.S.Ü., N.K., Analysis or Interpretation: A.D., A.K., N.K., Literature Search: S.U., Ö.U., N.K., A.D., Writing: A.K., S.S.Ü.

Conflict of Interest: No conflict of interest was declared by the authors.

Financial Disclosure: The authors declared that this study received no financial support.

\section{REFERENCES}

1. Sultan AA, Tata LJ, West J, Fiaschi L, Fleming KM, Nelson-Piercy C, et al. Risk factors for first venous thromboembolism around pregnancy: a population-based cohort study from the United Kingdom. Blood 2013;121:3953-61.

2. Berg Cl, Callaghan WM, Henderson Z, Syverson C. Pregnancyrelated mortality in the United States, 1998 to 2005. Obstet Gynecol 2010;116:1302-9.

3. Cantwell R, Clutton-Brock T, Cooper G, Dawson A, Drife J, Garrod D, et al. Saving Mothers' Lives: Reviewing maternal deaths to make motherhood safer: 2006-2008. The Eighth Report of the Confidential Enquiries into Maternal Deaths in the United Kingdom. BJOG 2011;118(Suppl.1):1-203

4. Marti C, John G, Konstantinides S, Combescure C, Sanchez O, Lankeit M, et al. Systemic thrombolytic therapy for acute pulmonary embolism: a systematic review and meta-analysis. Eur Heart J 2015;36:605-14.

5. Kearon C, Akl EA, Ornelas J, Blaivas A, Jimenez D, Bounameaux H, et al. Antithrombotic Therapy for VTE Disease: CHEST Guideline and Expert Panel Report. Chest 2016;149:315-52.

6. Agerstrand C, Abrams D, Biscotti M, Moroz L, Rosenzweig EB, D'Alton D, et al. Extracorporeal membrane oxygenation for cardiopulmonary failure during pregnancy and postpartum. Ann Thorac Surg 2016;102:774-9.

7. Martillotti G, Boehlen F, Robert-Ebadi H, Jastrow N, Righini M, Blondon $M$. Treatment options for severe pulmonary embolism during pregnancy and the postpartum period: a systematic review. J Thromb Haemost 2017;15:1942-50

8. MacLaren G, Combes A, Bartlett RH. Contemporary extracorporeal membrane oxygenation for adult respiratory failure: life support in the new era. Intensive Care Med 2012;38:210-20.

9. Frenckner B. Extracorporeal membrane oxygenation: a break-through for respiratory failure. J Intern Med 2015;278:586-98.

10. Pacheco LD, Saade GR, Hankins GDV. Extracorporeal membrane oxygenation (ECMO) during pregnancy and postpartum. Semin Perinatol 2018;42:21-5.

11. Moore SA, Dietl CA, Coleman DM. Extracorporeal life support during pregnancy. J Thorac Cardiovasc Surg 2016;151:1154-60.

12. Coughlin MA, Bartlett RH. Anticoagulation for extracorporeal life support: direct thrombin inhibitors and heparin. ASAIO J 2015;61:652-

13. Peek GL, Mugford M, Tiruvoipati R, Wilson A, Allen E, Thalanany MM, et al. Efficacy and economic assessment of conventional ventilatory support versus extracorporeal membrane oxygenation for severe adult respiratory failure (CESAR): a multicenter randomised controlledtrial. Lancet 2009;374:1351-63.

14. Zwischenberger JB, Lynch JE. Will CESAR answer the adult ECMO debate? Lancet 2009;374:1307-8.

15. Australia and New Zealand Extracorporeal Membrane Oxygenation (ANZ ECMO) Influenza Investigators, Davies A, Jones D, Bailey M, Beca J, Bellomo R, et al. Extracorporeal Membrane Oxygenation for 2009 Influenza A (H1N1) Acute Respiratory Distress Syndrome. JAMA 2009;302:1888-95.

16. Saad AF, Rahman M, Maybauer DM, Fraser JF, Constantine MM, Pacheco LD, et al. Extracorporeal membrane oxygenation in pregnant and postpartum women with H1N1-related acute respiratory distress syndrome: a systematic review and meta-analysis. Obstet Gynecol 2016;127:241-7. 
17. Chung M, Shiloh AL, Carlese A. Monitoring of the adultpatient on venoarterial extracorporeal membrane oxygenation. ScientificWorldJournal 2014;2014:393258.14.

18. Ali J, Vuylsteke A. Extracorporeal membrane oxygenation: indications, technique and contemporary outcomes. Heart 2019;105:1437-43.

19. Douflé G, Ferguson ND. Monitoring during extracorporeal membrane oxygenation. Curr Opin Crit Care 2016;22:230-8.

20. Imankulova B, Terzic M, Ukybassova T, Bapayeva G, Lesbekov T, Mustafinova G, et al. Repeated pulmonary embolism with cardiac arrest after uterine artery embolization for uterine arteriovenous malformation: A case report and literature review. Taiwan J Obstet Gynecol 2018;57:890-3.

21. Vashisht A, Katakam N, Kausar S, Patel N, Stratton J. Postnatal diagnosis of maternal congenital heart disease: missed opportunities. BMJ Case Rep 2015;2015:bcr2015209938.

22. UII C, Schildhauer TA, Strauch JT, Mügge A, Swol J. Veno-venous ECMO as a safe bridge to recovery in a patient with severe peripartum cardiomyopathy - learning from errors. Perfusion 2017;32:328-32.

23. Sharma NS, Wille KM, Bellot SC, Diaz-Guzman E. Modern use of extracorporeal life support in pregnancy and postpartum. ASAIO J 2015;61:110-4.

24. Kaur K, Bhardwaj M, Kumar P, Singhal S, Singh T, Hooda S. Amniotic fluid embolism. J Anaesthesiol Clin Pharmacol 2016;32:153-9.

25. Arlt M, Philipp A, lesalnieks I, Kobuch R, Graf BM. Successful use of a new hand-held ECMO system in cardiopulmonary failure and bleeding shock after thrombolysis in massive post-partal pulmonary embolism. Perfusion 2009;24:49-50.

26. Weinberg L, Kay C, Liskaser F, Jones D, Tay S, Jaffe S, et al. Successful treatment of peripartum massive pulmonary embolism with extracorporeal membrane oxygenation and catheter-directed pulmonary thrombolytic therapy. Anaesth Intensive Care 2011;39:48691.

27. Leeper WR, Valdis M, Arntfield R, Ray Guo L. Extracorporeal membrane oxygenation in the acute treatment of cardiovascular collapse immediately post-partum. Interact Cardiovasc Thorac Surg 2013;17:8989.

28. Ho YK, Wang CP, Wu YL, Lee TH, Ying TH, Lee MS. Pulmonary embolism after cesarean section and successful treatment with early application of extracorporeal membrane oxygenation system and anticoagulant agents. Taiwan J Obstet Gynecol 2014;53:273-5.

29. Fernandes P, Allen P, Valdis M, Guo L. Successful use of extracorporeal membrane oxygenation for pulmonary embolism, prolonged cardiac arrest, post-partum: a cannulation dilemma. Perfusion 2015;30:106-10.

30. Bataillard A, Hebrard A, Gaide-Chevronnay L, Casez M, Dessertaine G, Durand $\mathrm{M}$, et al. Extracorporeal life support for massive pulmonary embolism during pregnancy. Perfusion 2016;31:169-71.

31. McDonald C, Laurie J, Janssens S, Zazulak C, Kotze P, Shekar K. Successful provision of inter-hospital extracorporeal cardiopulmonary resuscitation for acute post-partum pulmonary embolism. Int J Obstet Anesth 2017;30:65-8.

32. Takacs ME, Damisch KE. Extracorporeal Life Support as Salvage Therapy for Massive Pulmonary Embolus and Cardiac Arrest in Pregnancy. J Emerg Med 2018;55:121-4.

33. Link MS, Berkow LC, Kudenchuk PJ, Halperin HR, Hess EP, Moitra VK, et al. Part 7: Adult Advanced Cardiovascular Life Support: 2015 American Heart Association Guidelines Update for Cardiopulmonary Resuscitation and Emergency Cardiovascular Care. Circulation 2015;132(18 Suppl 2):444-64.

34. Pacheco LD, Saade GR, Hankins GDV. Extracorporeal membrane oxygenation (ECMO) during pregnancy and postpartum. Semin Perinatol 2018;42:21-5. 